\title{
DOA estimation of wideband signals based on slice-sparse representation
}

\author{
Lu Gan* and Xiaoqing Wang
}

\begin{abstract}
In this article, the direction-of-arrival (DOA) estimation problem of wideband signal sources is studied. We pass the incident signals through a bank of narrowband filters to split the array outputs into several narrowband components. Then, a novel slice-sparse representation model of the joint narrowband array covariance data is proposed in the frequency domain to enforce joint sparsity in the concatenated covariance matrix of all frequencies. Based on the greed matching pursuit algorithm, a multiple measurement slices orthogonal matching pursuit algorithm is proposed to exploit the joint frequency processing in the case of wideband scenarios. The DOA estimation is achieved by joint processing of the array covariance data at different frequency bins. The estimated performance is compared with the representative DOA estimation methods. Simulation experiments are conducted to validate the effectiveness of the proposed method.
\end{abstract}

Keywords: Wideband signal, Direction-of-arrival, Slice-sparse representation, Joint frequency processing

\section{Introduction}

Due to the increase use of wideband signals in the fields of wireless communication system and radar, the problem of direction of arrival (DOA) estimation of wideband signals has been of considerable interest to the array signal processing in recent years. Many methods have been proposed to estimate the DOAs of wideband signals, among which the maximum-likelihood (ML) methods and subspace methods are most studied. The ML estimators show excellent performance but it needs multidimensional nonlinear global search [1]. The subspace methods, although not optimal, are computationally more attractive than ML methods. The subspace methods can be classified into two major categories: incoherent signal-subspace method (ISSM) [2] and coherent signal-subspace method (CSSM) [3-6]. These methods decompose the incident wideband signals into narrowband components by passing them through a bank of narrowband filters, and then obtain the DOA estimation incoherently or coherently. The ISSM incoherently constructs the final result by taking an average of different frequency bins. Although it

${ }^{*}$ Correspondence: ganlu@uestc.edu.cn

School of Electronic Engineering, University of Electronic Science and

Technology of China, Chengdu, Sichuan, 611731, China works well at high signal-to-noise ratio (SNR), its performance degrades greatly at low SNR or the SNR varies at different frequency bins because even a single outlier from one frequency bin can potentially lead to inaccurate estimate through the averaging processing. The CSSM surpasses the ISSM because of the capability to process the coherent sources by employing the spectral focusing technique [3]. However, the accuracy of preliminary DOA estimation which is required for the spectral focusing largely influences the performance of CSSM [6]. Therefore, we must explore better solutions for the problem of wideband DOA estimation.

In recent years, the sparse signal representation has attracted enormous attention. By now, there has been several articles addressed sparse representation (SR)-based DOA estimation [7-9]. Among them, the l1-SVD method proposed by Malioutov et al. [8] is a successful attempt. This method has also been extended to the wideband signals by treating each frequency band independently [8]. But, repeated using convex optimization technique leads to heavy computational burden in the multiple measurement vectors (MMV) scenario. The wideband covariance matrix sparse representation (W-CMSR) method presented by Liu and Haung [10] forms a new measurement vector by aligning the lower left triangular elements of the array output covariance matrix and reconstructs this

\section{是 Springer}

(c) 2013 Gan and Wang; licensee Springer. This is an Open Access article distributed under the terms of the Creative Commons Attribution License (http://creativecommons.org/licenses/by/2.0), which permits unrestricted use, distribution, and reproduction in any medium, provided the original work is properly cited. 
vector on an over-complete dictionary to realize wideband DOA estimation. But, it relies on some prior information such as signal modulation types and the pre-estimated signal power spectrum. These give rise to performance degradation when the incident signals share different types of modulations or signal power spectrums. Therefore, further efforts are required to derive integrative wideband DOA estimates method.

In this article, we still address the wideband DOA estimation problem by dividing the wideband data into narrowband counterparts. To work in the frequency domain, we propose a slice-SR model of the joint array covariance data that are stacked as a tensor matrix. Based on the greedy matching pursuit algorithm $[11,12]$, we propose a multiple measurement slices (MMS) orthogonal matching pursuit (MMS-OMP) algorithm. This algorithm processes the narrowband covariance data jointly to obtain the spatial-frequency spectrum. The proposed method and other representative methods are compared. The simulation results show that this algorithm has better noise robustness, higher resolution, and can resolve coherent sources.

This article contributes to the field of wideband DOA estimation in the following four aspects,

(1) We use the idea of joint processing of the narrowband components, so the spectral focusing is not introduced.

(2) The proposed method is based on the greedy pursuit algorithms so that, it is a low-complexity and high resolution estimator for the wideband signal sources.

(3) An important benefit that comes with our algorithm is the ability to incorporate prior information on the frequency spectra of the sources.

(4) Furthermore, we can allow the distance between adjacent array elements larger than the smallest half-wavelength with respect to the wideband source frequency, just like what W-CMSR has achieved.

The remainder of the article mainly consists of four sections. In Section 2, we describe the frequency domain model of wideband DOA estimation. In Section 3, we present the viewpoint of the SR of array output covariance vectors. Then, a concept of joint $K$-slice sparse is proposed for the stack operation of the MMS in the form of tensor matrix. Finally, we formulate the MMS-OMP algorithm in detail. Some numerical results are provided to demonstrate the performance and computational efficiency of MMS-OMP in Section 4. In Section 5, we make a conclusion.

\section{Data model}

For wideband signal sources, suppose that $K$ far-field wideband signals impinge onto an $N$-element array from the directions of $\theta_{1}, \theta_{2}, \ldots, \theta_{K}$ respectively, which are corrupted by additive complex Gaussian white noise. At time $t$, the data of the $n$th array element are collected by

$$
x_{n}(t)=\sum_{k=1}^{K} s_{k}\left(t-\tau_{n k}\right)+w_{n}(t) \quad n=1,2, \ldots, N
$$

where $s_{k}(t)$ is the waveform of the $k$ th source, $\tau_{n k}$ is the propagation delay of the $n$th element of the array with respect to the reference element, $w_{n}(t)$ is the zeromean complex additive Gaussian white noise. We assume that the incident signals and the additive noise are mutually independent. The number of sources $K$ can be estimated by Akaike information criterion (AIC) or minimum description length (MDL) criterion [13].

Then, the observation time $T$ is divided into $L$ subsegments. Each sub-segment has an observation time $T_{d}$, apparently, $T=L T_{d}$. We transform the $T_{d}$ received data of the $l$ th segment into the frequency domain resulting in $J$ non-overlapping narrowband components. We denote the frequency domain data at frequency $f_{j}$ by

$$
\begin{gathered}
\mathbf{X}_{l}\left(f_{j}\right)=\mathbf{A}\left(f_{j}\right) \mathbf{S}_{l}\left(f_{j}\right)+\mathbf{W}_{l}\left(f_{j}\right) \\
l=1,2, \ldots, L \quad j=1,2, \ldots, J
\end{gathered}
$$

where $\mathbf{X}_{l}\left(f_{j}\right) \in \mathbb{C}^{N \times 1}, \mathbf{S}_{l}\left(f_{j}\right) \in \mathbb{C}^{K \times 1}, \mathbf{W}_{l}\left(f_{j}\right) \in \mathbb{C}^{N \times 1}$ are derived from the discrete Fourier transform (DFT) of the received data, signals and noise, respectively.

The array manifold matrix with respect to the above wideband model is

$$
\mathbf{A}\left(f_{j}\right)=\left[\begin{array}{lllll}
\mathbf{a}_{1}\left(f_{j}\right) & \mathbf{a}_{2}\left(f_{j}\right) & \ldots & \mathbf{a}_{K}\left(f_{j}\right)
\end{array}\right] \in \mathbb{C}^{N \times K}
$$

Considering a uniform linear array (ULA), the array steering vector $\mathbf{a}_{k}\left(f_{j}\right)$ can be expressed as follows

$$
\mathbf{a}_{k}\left(f_{j}\right)=\left[\begin{array}{lllll}
e^{-j 2 \pi f_{j} \tau_{1 k}} & e^{-j 2 \pi f_{j} \tau_{2 k}} & \ldots & e^{-j 2 \pi f_{j} \tau_{N k}}
\end{array}\right]
$$

where $\tau_{n k}=\frac{1}{c}(n-1) d \sin \left(\theta_{k}\right), d$ is the distance between adjacent array element, $c$ is the velocity of wave propagation.

Equation (2) is the frequency domain model of wideband signal sources. It is similar to time domain model of narrowband sources in the form. The estimated array covariance matrix at frequency $f_{j}$ is given by

$$
\hat{\mathbf{R}}\left(f_{j}\right)=\frac{1}{L} \sum_{l=1}^{L} \mathbf{X}_{l}\left(f_{j}\right) \mathbf{X}_{l}^{H}\left(f_{j}\right), \quad j=1,2, \ldots, J
$$

where $\hat{\mathbf{R}}\left(f_{j}\right) \in \mathbb{C}^{N \times N},[\cdot]^{H}$ denotes the complex conjugate transpose operator. The wideband DOA estimation method proposed in next section can be derived from $\mathrm{SR}$ of the array output covariance vectors in $\hat{\mathbf{R}}\left(f_{j}\right)$. 


\section{Proposed method}

\subsection{SR of the array covariance vectors}

For the frequency $f_{j}$, (5) can be reformulated as follows

$$
\hat{\mathbf{R}}\left(f_{j}\right)=\mathbf{A}\left(f_{j}\right) \hat{\mathbf{R}}_{S}\left(f_{j}\right) \mathbf{A}^{H}\left(f_{j}\right)+\sigma_{W}^{2}\left(f_{j}\right) \mathbf{I}
$$

where $\hat{\mathbf{R}}_{S}\left(f_{j}\right)$ is the signal covariance matrix, $\sigma_{W}^{2}\left(f_{j}\right)$ is the noise power, $\mathbf{I}$ is an $N \times N$ identity matrix. In order to a convenient derivation, we temporarily neglect the noise item. Then we have

$$
\hat{\mathbf{R}}\left(f_{j}\right)=\mathbf{A}\left(f_{j}\right)\left[\hat{\mathbf{R}}_{S}\left(f_{j}\right) \mathbf{A}^{H}\left(f_{j}\right)\right]
$$

According to SR theory [14], it is obviously that (7) can be rewritten as $\hat{\mathbf{R}}\left(f_{j}\right)=\mathbf{A}\left(f_{j}\right) \mathbf{G}$, where $\mathbf{G}=$ $\hat{\mathbf{R}}_{S}\left(f_{j}\right) \mathbf{A}^{H}\left(f_{j}\right) \in \mathbb{C}^{K \times N}, \mathbf{A}\left(f_{j}\right)=\left[\begin{array}{llll}\mathbf{a}_{1}\left(f_{j}\right) & \mathbf{a}_{2}\left(f_{j}\right) & \ldots & \mathbf{a}_{K}\left(f_{j}\right)\end{array}\right]$. We represent $\hat{\mathbf{R}}\left(f_{j}\right)$ in the form of column vectors as $\hat{\mathbf{R}}\left(f_{j}\right)=\left[\begin{array}{llll}\hat{\mathbf{r}}_{1}\left(f_{j}\right) & \hat{\mathbf{r}}_{2}\left(f_{j}\right) & \ldots & \hat{\mathbf{r}}_{N}\left(f_{j}\right)\end{array}\right]$. So each column vector in $\hat{\mathbf{R}}\left(f_{j}\right)$ can be regarded as the linear combination of the array steering vectors in $\mathbf{A}\left(f_{j}\right)$, i.e.,

$$
\hat{\mathbf{r}}_{n}\left(f_{j}\right)=\sum_{k=1}^{K} g_{k n} \mathbf{a}_{k}\left(f_{j}\right)
$$

where $g_{k n}$ is the $k$ th row $n$th column element of G. To cast this problem as a SR problem, we introduce an overcomplete representation $\boldsymbol{\Phi}_{j}$ in terms of all possible DOAs. Then, let $\left\{\tilde{\theta}_{1}, \tilde{\theta}_{2}, \ldots, \tilde{\theta}_{N_{s}}\right\}$ be a sampling grid of all DOAs of interest. The number of potential DOAs $N_{s}$ will typically be much greater than the number of sources $K$. Then, we construct the over-complete basic matrix composed of steering vectors corresponding to each potential DOA as its columns

$$
\boldsymbol{\Phi}_{j}=\left[\begin{array}{lllll}
\mathbf{a}\left(\tilde{\theta}_{1}, f_{j}\right) & \mathbf{a}\left(\tilde{\theta}_{2}, f_{j}\right) \quad \ldots & \mathbf{a}\left(\tilde{\theta}_{N_{s}}, f_{j}\right)
\end{array}\right]
$$

So (8) can be represented as

$$
\hat{\mathbf{r}_{n}}\left(f_{j}\right)=\sum_{n_{s}=1}^{N_{s}} b_{n_{s}} \mathbf{a}\left(\tilde{\theta}_{n_{s}}, f_{j}\right)
$$

where the linear combination coefficient $b_{n_{s}}$ satisfy

$$
b_{n_{s}}= \begin{cases}g_{k n} & \tilde{\theta}_{n_{s}}=\theta_{k} \\ 0 & \tilde{\theta}_{n_{s}} \neq \theta_{k}\end{cases}
$$

So $\hat{\mathbf{r}}_{n}\left(f_{j}\right)$ is the linear combination of the range space of $\boldsymbol{\Phi}_{j}$. We reformulate (10) as the form of matrices, then we obtain

$$
\hat{\mathbf{r}_{n}}\left(f_{j}\right)=\boldsymbol{\Phi}_{j} \mathbf{b}_{n, j} \quad n=1,2, \ldots, N
$$

where $\mathbf{b}_{n, j}$ is the $N_{s} \times 1$ representation coefficient vector with respect to the above over-complete basis at frequency $f_{j}$. Since $\boldsymbol{\Phi}_{j}$ has a nontrivial nullspace, many solutions of $\mathbf{b}_{n, j}$ can fit $\hat{\mathbf{r}}_{n}\left(f_{j}\right)$ well. To make $\mathbf{b}_{n, j}$ unique $K$ sparse solution, we should impose the sparsity of the signals relative to the whole spatial domain. If $\left\{\tilde{\theta}_{1}, \tilde{\theta}_{2}, \ldots, \tilde{\theta}_{N_{s}}\right\}$ are dense enough, some $K$ angles in $\left\{\tilde{\theta}_{1}, \tilde{\theta}_{2}, \ldots, \tilde{\theta}_{N_{s}}\right\}$ can be expected to be very close (or even equal) to the actual $\left\{\theta_{1}, \theta_{2}, \ldots, \theta_{K}\right\}$, so an ideal $\mathbf{b}_{n, j}$ should be the vector with all elements zero except for the $K$ elements associated with these $K$ angles, which are related to the DOAs of the signals [14].

Then, we rewrite the model in (7) in the form of matrix as follows

$$
\hat{\mathbf{R}}\left(f_{j}\right)=\boldsymbol{\Phi}_{j} \mathbf{B}_{j}
$$

where $\mathbf{B}_{j}=\left[\mathbf{b}_{1, j}, \mathbf{b}_{2, j}, \ldots, \mathbf{b}_{N, j}\right] \in \mathbb{C}^{N_{s} \times N}$. It is clear that the ideal $\mathbf{b}_{n, j}, \quad n=1,2, \ldots, N$ should share the same sparse structure, and the nonzero elements of each ideal $\mathbf{b}_{n, j}$ should occur in the same rows of $\mathbf{B}_{j}$. In other words, only $K$ rows of $\mathbf{b}_{n, j}$ are nonzero. Such a matrix is called joint $K$-rows sparse [12]. We call $\hat{\mathbf{r}}_{n}\left(f_{j}\right), \quad n=1,2, \ldots, N$ as MMV.

Slices are the two-dimensional sections of a tensor matrix. Figures 1 and 2 show the horizontal, lateral, and frontal slices of a tensor matrix [15]. The above analyzes the SR of the array output covariance vectors at frequency $f_{j}$. For all narrowband data of every frequency bins, we have MMS, which consist of corresponding array output covariance vectors. Just as shown in Figure 1, the MMS with respect to corresponding frequency bins are stacked up as frontal slices. As a result, $\mathbf{B}_{j}, j=1,2, \ldots, J$ are stacked up in the form of three-dimensional (3-D) tensor matrix $\mathcal{B}$. The new tensor matrix $\mathcal{B} \in \mathbb{C}^{N_{s} \times N \times J}$ illustrated in Figure 3 has a property of nonzero elements occupying only $K$ horizontal slices, so that it is called joint $K$-slice sparse. Therefore, the DOA estimation problem can be posed as the MMS problem of finding a slice-sparse tensor matrix $\mathcal{B}$ to represent the $3-\mathrm{D}$ measurement matrix $\mathcal{R} \in \mathbb{C}^{N \times N \times J}$ under the tensor basic matrix $\boldsymbol{\Phi} \in \mathbb{C}^{N \times N_{s} \times J}$.

For the above joint $K$-slice sparse model, we formulate our algorithm based on the fundamental OMP algorithm. In order to facilitate the presentation before introducing our algorithm, we define some concepts as follows.

(1) $\|\cdot\|_{F}^{2}$ and $\|\cdot\|^{2}$ denote the Frobenius norm for a matrix and the Euclidean norm for a vector;

(2) $\mathbf{R}_{j}^{(i)}, j=1,2, \ldots, J$ illustrated in Figure 2 denotes the residual matrices after $i$ th iteration, where $\mathbf{R}_{j}^{(0)}=\hat{\mathbf{R}}\left(f_{j}\right), j=1,2, \ldots, J$, the $n$th column of $\mathbf{R}_{j}^{(i)}$ is denoted by $\mathbf{r}_{n, j}^{(i)}$;

(3) $S_{i}=\left\{s_{1}, s_{2}, \ldots, s_{i}\right\}, S_{0}=\emptyset$. We denote this subset which stores the indices $s_{i}$ of the $i$ lateral slices selected from the tensor basic matrix $\boldsymbol{\Phi}$;

(4) $\boldsymbol{\Omega}_{i}=\left[\boldsymbol{\psi}_{s_{1}}, \boldsymbol{\psi}_{s_{2}}, \ldots, \boldsymbol{\psi}_{s_{i}}\right], \boldsymbol{\Omega}_{0}=\emptyset$. This tensor matrix named atom set stores the selected lateral slices from $\boldsymbol{\Phi}$, where $\boldsymbol{\psi}_{s_{i}}$ showed in Figure 2 denotes the selected lateral slice;

(5) $P_{\boldsymbol{\Omega}_{j}^{i}}$ represents the orthogonal projection matrix onto the range space of $\boldsymbol{\Omega}_{j}^{i}$, where $\boldsymbol{\Omega}_{j}^{i}$ is the $j$ th frontal slice 


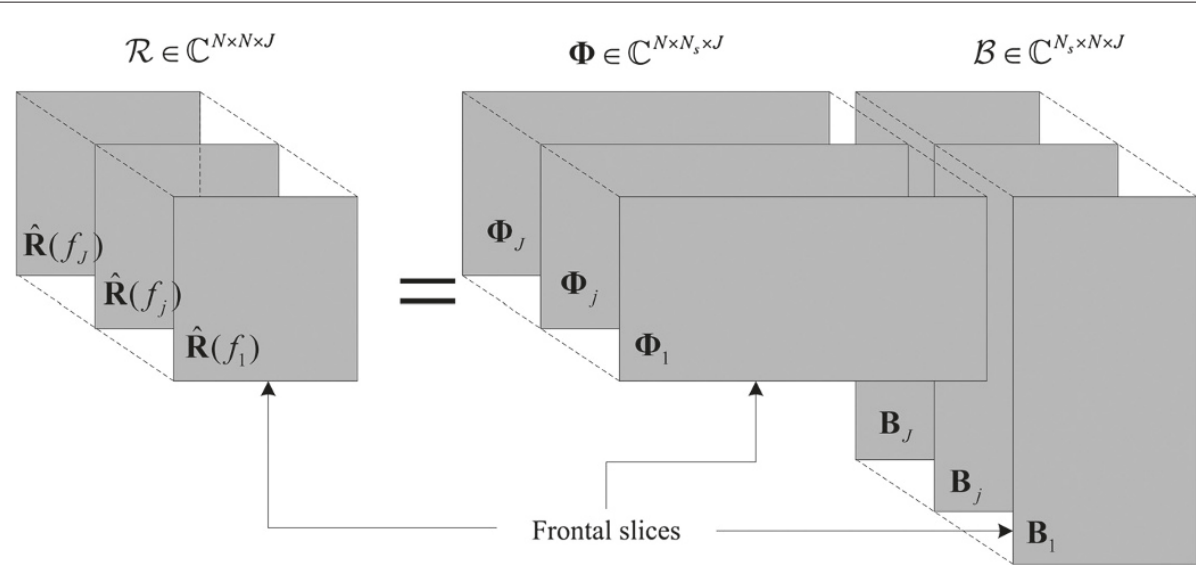

Figure 1 The stacking operation of the array covariance matrices at different frequency bins. The MMS with respect to corresponding frequency bins are stacked up as frontal slices.

which is related to the frequency $f_{j}$. Its orthogonal complement is $P_{\boldsymbol{\Omega}_{j}^{i}}^{\perp}=\left(\mathbf{I}-P_{\boldsymbol{\Omega}_{j}^{i}}\right), P_{\boldsymbol{\Omega}_{j}^{0}}=\mathbf{0}, P_{\boldsymbol{\Omega}_{j}^{0}}^{\perp}=\mathbf{I}$.

\subsection{OMP algorithm}

Orthogonal matching pursuit algorithm [11] can process the underdetermined equation solution in (12), i.e., $\hat{\mathbf{r}}_{n}\left(f_{j}\right)=\boldsymbol{\Phi}_{j} \mathbf{b}_{n, j}$. Since $\mathbf{b}_{n, j}$ has only $K$ nonzero components, the vector $\hat{\mathbf{r}}_{n}\left(f_{j}\right)=\boldsymbol{\Phi}_{j} \mathbf{b}_{n, j}$ is a linear combination of $K$ columns from $\boldsymbol{\Phi}_{j}$. To identify the ideal $\mathbf{b}_{n, j}$, we need to determine which columns of $\boldsymbol{\Phi}_{j}$ participate in the measurement vector $\hat{\mathbf{r}}_{n}\left(f_{j}\right)$. The idea behind the algorithm is to pick columns in a greedy fashion. At $i$ th iteration, we choose the column $s_{i}$ of $\boldsymbol{\Phi}_{j}$ that is most strongly correlated with the remaining part of $\hat{\mathbf{r}}_{n}\left(f_{j}\right)$ by

$$
s_{i}=\arg \max _{s}\left\|\boldsymbol{\Phi}_{j}^{H} \overline{\mathbf{r}}_{j}^{(i-1)}\right\|^{2}, \quad s=1,2, \ldots, N_{s}
$$

where $\overline{\mathbf{r}}_{j}^{(i-1)}$ is the $(i-1)$ th residual and $\overline{\mathbf{r}}_{j}^{(0)}=\hat{\mathbf{r}}_{n}\left(f_{j}\right)$. Then we subtract off its contribution to $\hat{\mathbf{r}}_{n}\left(f_{j}\right)$ by

$$
\overline{\mathbf{r}}_{j}^{(i)}=\hat{\mathbf{r}}_{n}\left(f_{j}\right)-\boldsymbol{\Omega}_{i} \hat{\mathbf{b}}_{n, j}^{(i)}
$$

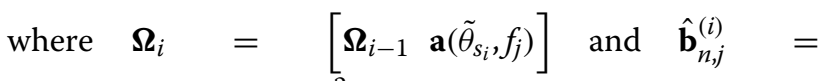
$\arg \min _{\mathbf{b}}\left\|\hat{\mathbf{r}}_{n}\left(f_{j}\right)-\boldsymbol{\Omega}_{i} \mathbf{b}\right\|^{2}$. Then, we iterate on the residual. One hopes that, after $K$ iterations, the algorithm will have identified the correct set of columns.

\subsection{MMS-OMP algorithm}

Considering the standard OMP algorithm [11], the sparse solution can be found by building up a small subset of column vectors selected from $\boldsymbol{\Phi}_{j}$ (we denote it with support set) to represent $\hat{\mathbf{r}}_{n}\left(f_{j}\right)$ sequentially. The selection of a nonzero row of $\mathbf{b}_{n, j}$ equals to selecting a column of $\boldsymbol{\Phi}_{j}$. In the joint $K$-slice sparse scenario, we use an extensional criterion to select the support set. Consequently, favorable performance and lower computational complexity are achieved. So our method presented in the followings is motivated by and is extensions of method developed for $J=1$ and $n=1$.

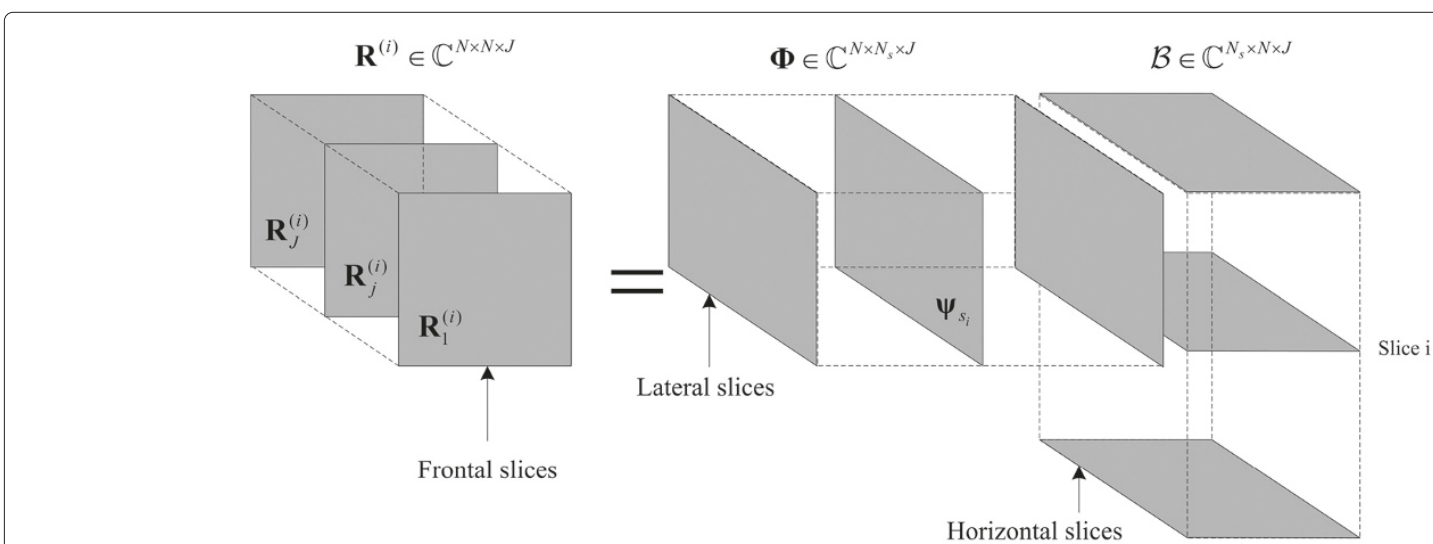

Figure 2 The illustration of the denoted concepts for MMS-OMP algorithm. The new residual are stacked up as frontal slices. The selected lateral slice is $\boldsymbol{\psi}_{s_{i}}$ at ith iteration. The corresponding ith horizontal slice is Slice $i$. 


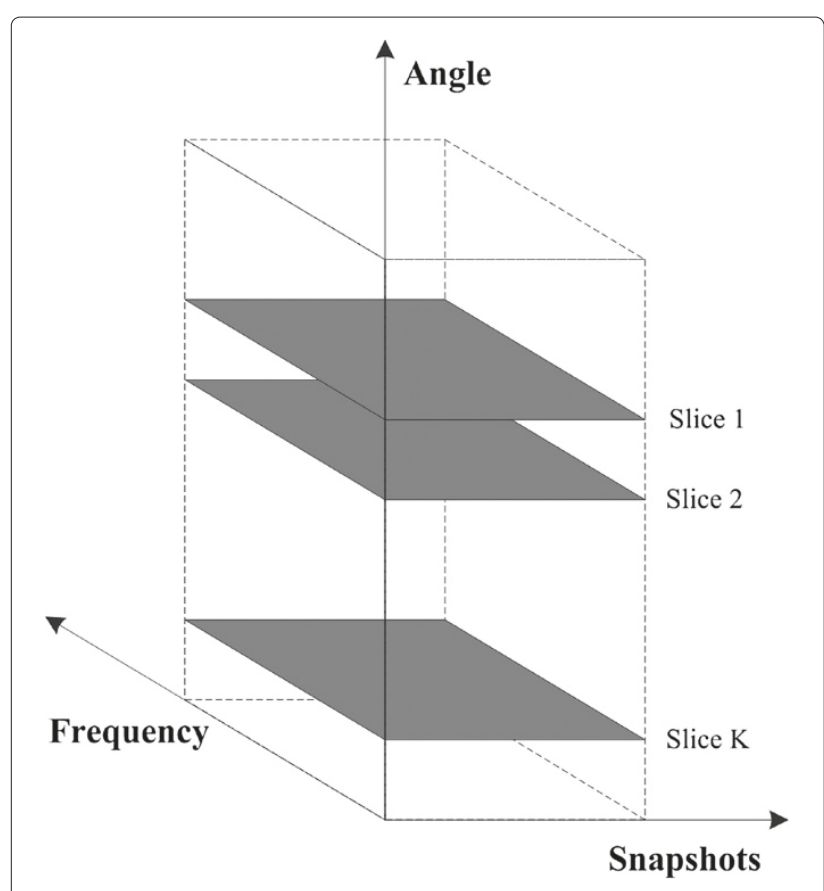

Figure 3 The slice-sparse architecture of the sparse tensor matrix $\mathcal{B}$. The sparse tensor matrix $\mathcal{B}$ has a property of nonzero elements occupying only $K$ horizontal slices.

In the MMS-OMP algorithm, we first find the lateral slice in the tensor matrix $\boldsymbol{\Phi}$, which is best aligned with the MMV (i.e., $\left.\mathbf{r}_{n, j}^{(i)}, j=1,2, \ldots, J, \quad n=1,2, \ldots, N\right)$ comprising the lateral slices of $\mathbf{R}^{(0)}=\mathcal{R}$, and this is denoted by $\boldsymbol{\psi}_{s_{1}}$. Then, the projection of $\mathbf{R}_{j}^{(0)}, j=1,2, \ldots, J$ along the direction $\psi_{s_{1}}, j$ is removed from $\mathbf{R}_{j}^{(0)}$, and the residual $\mathbf{R}^{(1)}$ is obtained by stacking each residual $\mathbf{R}_{j}^{(1)}$ from front to back. Next, the lateral slice $\boldsymbol{\psi}_{s_{2}}$ in $\boldsymbol{\Phi}$, which is best aligned with $\mathbf{R}^{(1)}$, is found, and a new residual $\mathbf{R}^{(2)}$ is formed. The algorithm proceeds by sequentially choosing the lateral slices that best matches the residual matrix. Now, we go into the detail of the MMS-OMP algorithm by looking at the $i$ th iteration.

In the $i$ th iteration, we find the slice matrix most closely aligned with the residual $\mathbf{R}^{(i-1)}$ by examining the total matching error

$$
\Delta_{s}^{(i)}=\sqrt{\sum_{j=1}^{J}\left|\Delta_{s, j}^{(i)}\right|^{2}}, \quad s=1,2, \ldots, N_{s}
$$

where $\Delta_{s, j}^{(i)}=P_{\mathbf{a}_{s, j}}^{\perp} \mathbf{r}_{j}^{(i-1)}$ is the $j$ th sub matching error, $\mathbf{a}_{s, j}$ is the $s$ th column vector of $\boldsymbol{\Phi}_{j}$. The vector which minimizes the Frobenius norm of the $j$ th sub matching error is selected, i.e.,

$$
\begin{aligned}
\left\|\Delta_{s, j}^{(i)}\right\|_{F}^{2} & =\operatorname{Tr}\left(\left(\Delta_{s, j}^{(i)}\right)^{H} \Delta_{s, j}^{(i)}\right) \\
& =\operatorname{Tr}\left(\left(P_{\mathbf{a}_{s, j}}^{\perp} \mathbf{R}_{j}^{(i-1)}\right)^{H} P_{\mathbf{a}_{s, j}}^{\perp} \mathbf{R}_{j}^{(i-1)}\right) \\
& =\left\|\mathbf{R}_{j}^{(i-1)}\right\|_{F}^{2}-\operatorname{Tr}\left(\left(\mathbf{R}_{j}^{(i-1)}\right)^{H} P_{\mathbf{a}_{s, j}} \mathbf{R}_{j}^{(i-1)}\right)
\end{aligned}
$$

The minimization is achieved by maximizing the second term in the above expression. $P_{\mathbf{a}_{s, j}}=\mathbf{a}_{s, j} \mathbf{a}_{s, j}^{H}$ is employed to select the index of lateral slice as

$$
s_{i, j}=\arg \max _{s}\left\|\mathbf{u}_{s, j}\right\|^{2}, \quad s=1,2, \ldots, N_{s}
$$

where $\mathbf{u}_{s, j}^{H}=\mathbf{a}_{s, j}^{H} \mathbf{R}_{j}^{(i-1)}$.

According to the joint $K$-slice sparse model, different sub matching error should share the uniform result of minimization. If all the minimization of sub matching errors $\Delta_{s, j}^{(i)}$ is achieved, the total matching error is achieved, too. Then, we modify (18) as follows

$$
s_{i}=\arg \max _{s}\left\|\sqrt{\sum_{j=1}^{J}\left|\mathbf{u}_{s, j}\right|^{2}}\right\|^{2}, \quad s=1,2, \ldots, N_{s}
$$

The support set is update by $S_{i}=S_{i-1} \cup\left\{s_{i}\right\}$ and $\boldsymbol{\Omega}_{i}=\left[\boldsymbol{\Omega}_{i-1}, \boldsymbol{\psi}_{s_{i}}\right]$. The new residual $\mathbf{R}_{j}^{(i)}$ is respectively computed as

$$
\mathbf{R}_{j}^{(i)}=P_{\mathbf{\Omega}_{j}^{i}}^{\perp} \mathbf{R}_{j}^{(i-1)}=\mathbf{R}_{j}^{(i-1)}-P_{\mathbf{\Omega}_{j}^{i}} \mathbf{R}_{j}^{(i-1)}, j=1,2, \ldots, J
$$

where $P_{\boldsymbol{\Omega}_{j}^{i}}$ is the projection matrix of $\boldsymbol{\Omega}_{j}^{i}$. The MMS-OMP algorithm is mainly defined by (19) and (20). The main steps of the MMS-OMP algorithm can be described as follows.

\section{The MMS-OMP algorithm.}

Input:

Tensor matrix $\mathcal{R}$;

Tensor basic matrix $\boldsymbol{\Phi}$;

Iterations $K$.

Output:

Tensor matrix $\hat{\mathcal{B}}$,

The spatial spectrum $P_{m m s \_o m p}$.

Initialization:

$\mathbf{R}^{(0)}=\mathcal{R}$ Initial residue;

$S_{0}=\emptyset$ Initial support set;

$\boldsymbol{\Omega}_{0}=\emptyset$ Initial atom set;

$i=0$ Initial iteration counter.

Iteration:

(1) Solve the optimization problem

$$
s_{i}=\arg \max _{s}\left\|\sqrt{\sum_{j=1}^{J}\left|\mathbf{u}_{s, j}\right|^{2}}\right\|^{2}, s=1,2, \ldots, N_{s} .
$$


(2) Update the support set and the selected slices $S_{i}=S_{i-1} \cup\left\{s_{i}\right\}$ and $\boldsymbol{\Omega}_{i}=\left[\boldsymbol{\Omega}_{i-1}, \boldsymbol{\psi}_{s_{i}}\right]$.

(3) Calculate the new residue respectively $\mathbf{R}_{j}^{(i)}=P_{\boldsymbol{\Omega}_{j}^{i}}^{\perp} \mathbf{R}_{j}^{(i-1)}=\mathbf{R}_{j}^{(i-1)}-P_{\boldsymbol{\Omega}_{j}^{i}} \mathbf{R}_{j}^{(i-1)}, j=1,2, \ldots, J$.

(4) If $i<K, i=i+1$ and return to step 1 ), otherwise quit the iteration.

(5) Estimate the tensor matrix $\mathcal{B}$ $\hat{\mathcal{B}}_{: j:}=\arg \min _{\mathcal{B}_{: j:}}\left\|\mathbf{R}_{j}^{(0)}-\boldsymbol{\Omega}_{j}^{(K)} \hat{\mathcal{B}}_{: j:}\right\|_{F}^{2}, j=1,2, \ldots, J$.

(6) Finally, Obtain the spatial spectrum by $P_{\text {mms_omp }}\left(n_{s}\right)=\| \hat{\mathcal{B}}_{n_{s}:: \|_{F}}^{2}, \quad n_{s}=1,2, \ldots, N_{s}$.

We will quit the iteration if halting condition is true. For example, we just need $K$ iteration, since the number of incident signals is known. The algorithm finally obtains the support set $S_{K}$. On one hand, we can transform it into angle value directly. The $k$ th DOA can be computed by $\theta_{k}=\theta_{\min }+\left(s_{k}-1\right) h$, where $\theta_{\min }$ and $h$ are the minimum angle and the step size in the direction grid, respectively. On the other hand, the estimated tensor matrix $\hat{\mathcal{B}}$ can be employed to form the spatial-frequency spectrum, and then the spatial spectrum is obtained via the average of each frequency counterpart, i.e.,

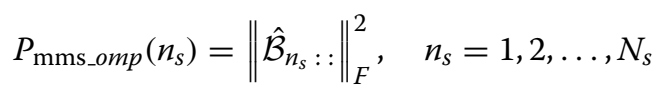

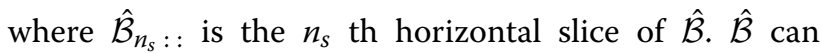
be obtained by stacking up the frontal slices $\hat{\mathcal{B}}_{: j:, j=}=$ $1,2, \ldots, J$, which can be calculated by

$$
\hat{\mathcal{B}}_{: j:}=\arg \min _{\hat{\mathcal{B}}_{: j:}}\left\|\mathbf{R}_{j}^{(0)}-\boldsymbol{\Omega}_{j}^{(K)} \hat{\mathcal{B}}_{: j:}\right\|_{F}^{2}, \quad j=1,2, \ldots, J
$$

Then, the DOAs can be found by searching the spectral peak.

\section{Simulation results}

In this section, some simulations are carried out to check the performance of MMS-OMP in wideband DOA estimation. The proposed method is compared with the previous methods, including two conventional subspace algorithm-MUSIC [2], CSSM [5] and a kind of SR algorithm-W-CMSR [10], and W-CMSR(SK) [10] represents that the prior spectrum information on W-CMSR is known.

In the simulation I, an intuitionistic spectrum comparison is given between MMS-OMP and previous methods. Assume that there are two far-field uncorrelated wideband signal sources impinging onto an ULA with $N=10$ array elements from the directions of $10^{\circ}$ and $30^{\circ}$. The incident signals are BPSKs with the central frequency $10 \mathrm{MHz}$ and the bandwidth $4 \mathrm{MHz}$, so that the ratio of bandwidth to central frequency is $40 \%$. The distance between adjacent array elements equals the half-wavelength with respect to the highest signal frequency. The distance between adjacent array elements equals the half-wavelength with respect to the highest signal frequency. We take $N_{s}=360$ by searching the angle space from $-90^{\circ}$ to $89.5^{\circ}$ with step size $0.5^{\circ}$. The SNR is set to $-10 \mathrm{~dB}$ and the number of time domain snapshots is set to 80 . We do the temporal-spectral transformation and collect the spectral snapshots by decomposing the sources into 80 frequency bins for MUSIC, CSSM and MMS-OMP. We retain the whole spatial-frequency spectrum of these two methods (MUSIC and MMS-OMP) in Figure 4a,b to give a more composite view of these methods. The 3-D spectrum of MUSIC shows that the peak locations and amplitudes for different frequency points are quite different, due to the fact that it treats each frequency independently. To mitigate this artifact, the MMS-OMP algorithm can incorporate a prior on the continuity of the frequency spectrum of the sources because of the joint frequency processing technique. Then, we average the 3-D spectrum of MUSIC and MMS-OMP in the frequency domain and reprint the corresponding 2-D spectrum in Figure 4c for a more convenient comparison with CSSM and W-CMSR. The results indicate that MUSIC, CSSM, W-CMSR all cannot express angular distribution correctly and pseudo-peaks exist in the spectrum of the three while the MMS-OMP algorithm can gain two correct peaks at $10^{\circ}$ and $30^{\circ}$.

In the simulation II, we examine the performance of MMS-OMP in the presence of spatial aliasing. The interspacing of the array is enlarged to 1.5 times wavelength associated with the highest signal frequency. 1,200 time domain snapshots are collected and the SNR is $10 \mathrm{~dB}$. Figure 5 shows that MUSIC and CSSM suffer from severe aliasing, and are unable to give any meaningful results. MMS-OMP can suppress the aliasing effects by employing the joint frequency processing technique over the whole frequency range.

In the simulation III, we consider the root mean square error (RMSE) of MMS-OMP and several existing DOA estimation methods, in the statistical sense for signals of various snapshots and SNRs. The curves were obtained by averaging the results of 500 Monte-Carlo simulations. We assume that two BPSK signals with bandwidth of $40 \%$ impinge onto the 10-ULA and the direction of the signal is fixed at $10^{\circ}$ and $30^{\circ}$. Firstly, we compare the RMSE of the four methods in the case of collecting 1,200 snapshots. We can see that the RMSE of the proposed method are smaller than the others in different SNRs indicated in Figure 6. Although W-CMSR(SK) makes full use of prior spectrum information such as code-rate for BPSK, its RMSE is higher than MMS-OMP. These confirm that our method is a more robust algorithm against DOA estimation error. Secondly, we give a comparison of the RMSE 
(a)

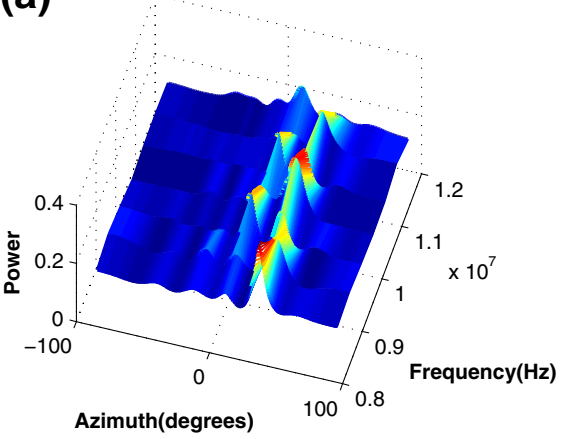

(b)

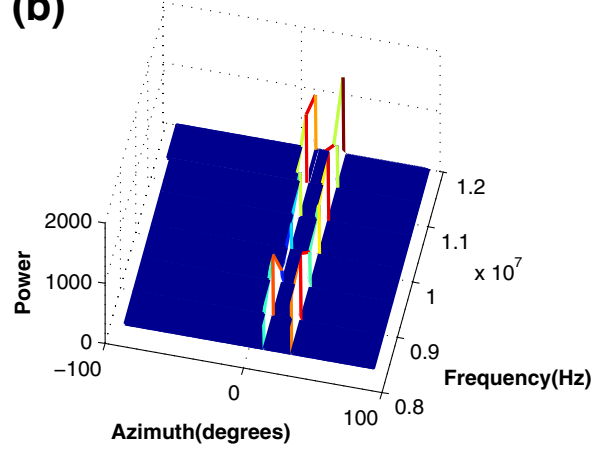

(c)

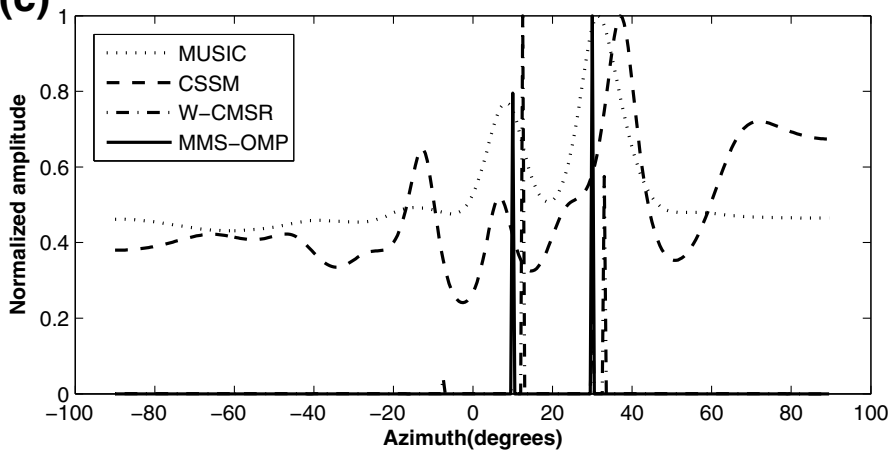

Figure 4 The intuitionistic spatial-frequency spectrum comparison of the four algorithms. (a) The 3-D spatial-frequency spectrum of MUSIC algorithm. (b) The 3-D spatial-frequency spectrum of MMS-OMP algorithm. (c) The 2-D normalized spatial spectrum of MUSIC, CSSM, W-CMSR and MMS-OMP algorithm. An intuitionistic spectrum comparison is given in these figures. The scenario is composed by two sources located at $\theta_{1}=10^{\circ}$ and $\theta_{2}=30^{\circ}, N=10$ sensors, $T=80$ time snapshots, and $S N R=-10 \mathrm{~dB}$. The ratio of bandwidth to central frequency is $40 \%$.

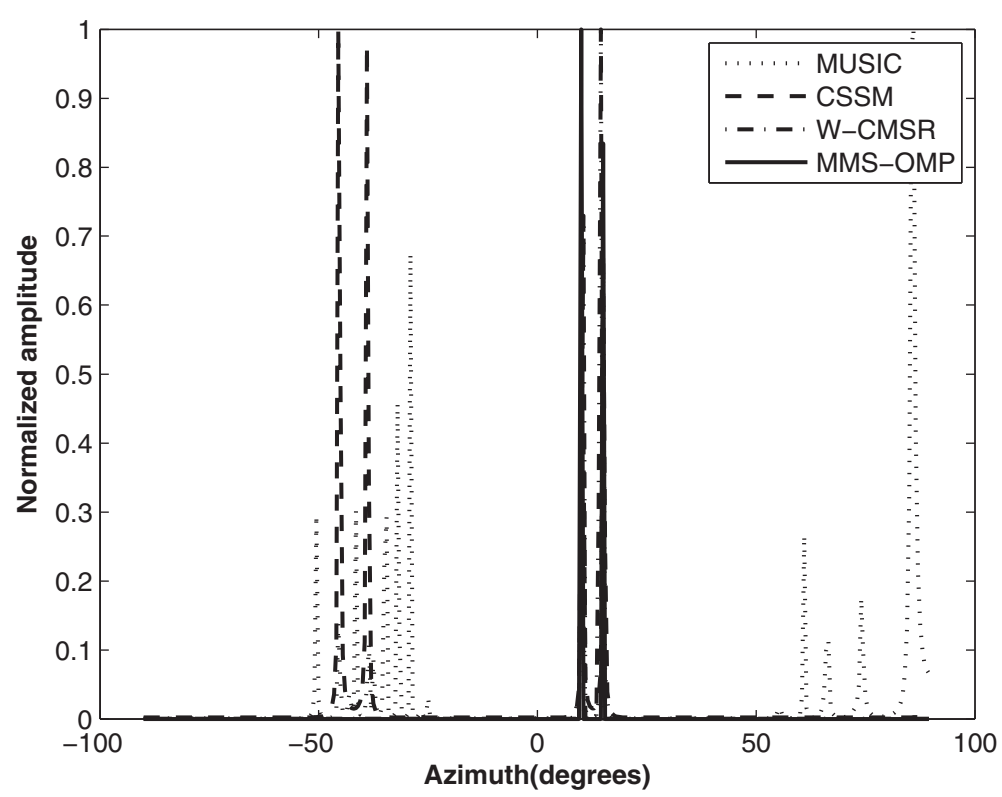

Figure 5 The 2-D normalized spatial spectrum of the four algorithms when the inter-spacing of array equals 1.5 times the smallest wavelength. The scenario is composed by two sources located at $\theta_{1}=10^{\circ}$ and $\theta_{2}=30^{\circ}, \mathrm{N}=10$ sensors, $T=1200$ time snapshots, and SNR $=$ $10 \mathrm{~dB}$. The ratio of bandwidth to central frequency is $40 \%$. This figure shows the estimated performance of the proposed method when the array inter-spacing is enlarged to 1.5 times wavelength associated with the highest signal frequency. 


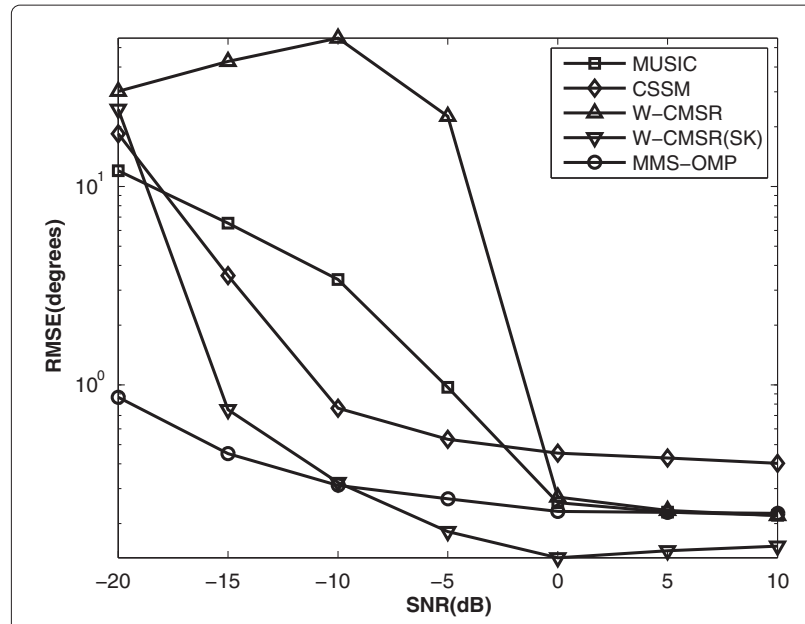

Figure 6 The RMSE of the compared algorithms versus SNR. The RMSE is defined by the formula

RMSE $=\frac{1}{K} \sum_{k=1}^{K} \sqrt{\frac{1}{\text { Mon }} \sum_{\text {mon }=1}^{\text {Mon }}\left(\hat{\theta}_{k, \text { mon }}-\theta_{k}\right)^{2}}$, where Mon is the number of Monte-Carlo simulations, $\hat{\theta}_{k, \text { mon }}$ is the $k$ th DOA estimated value of the month simulation. The settings are: $\theta_{1}=10^{\circ}, \theta_{2}=30^{\circ}, N=10$ sensors, $T=1,200$ time snapshots. The number of Monte-Carlo simulations is $\mathrm{Mon}=500$.

by fixing the SNR at $10 \mathrm{~dB}$ when the snapshots vary from 320 to 1,280 . Figure 7 shows that our method also has a good adaptability to snapshots.

Simulation IV compares the estimated probability of the four algorithms with the change of SNR, which is derived from 500 Monte-Carlo simulations whose condition is the same as simulation III. Our method gives higher estimated probability than other algorithm when the SNR

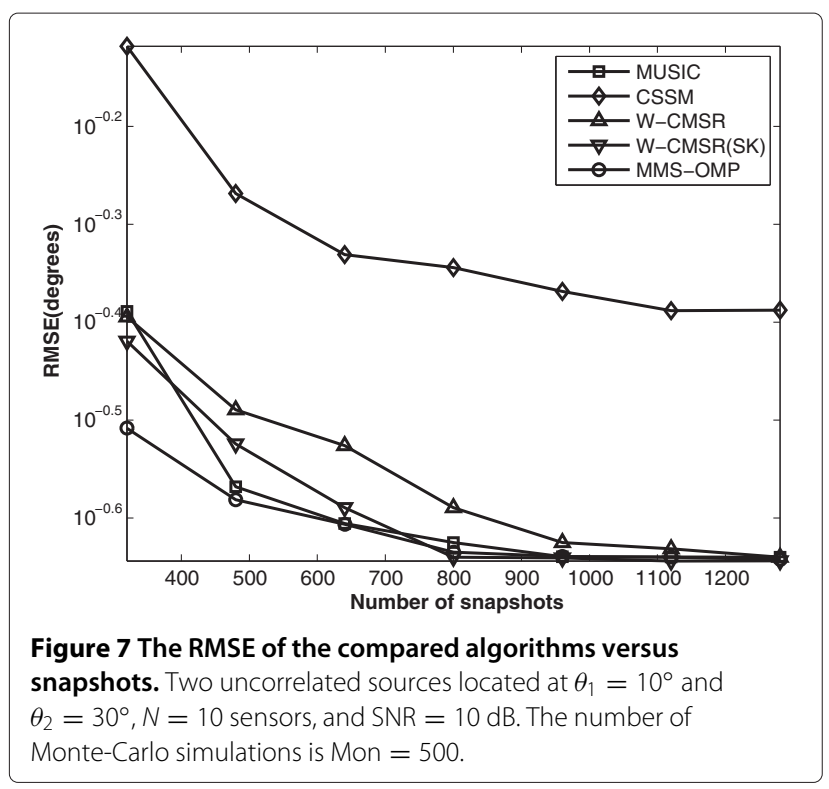

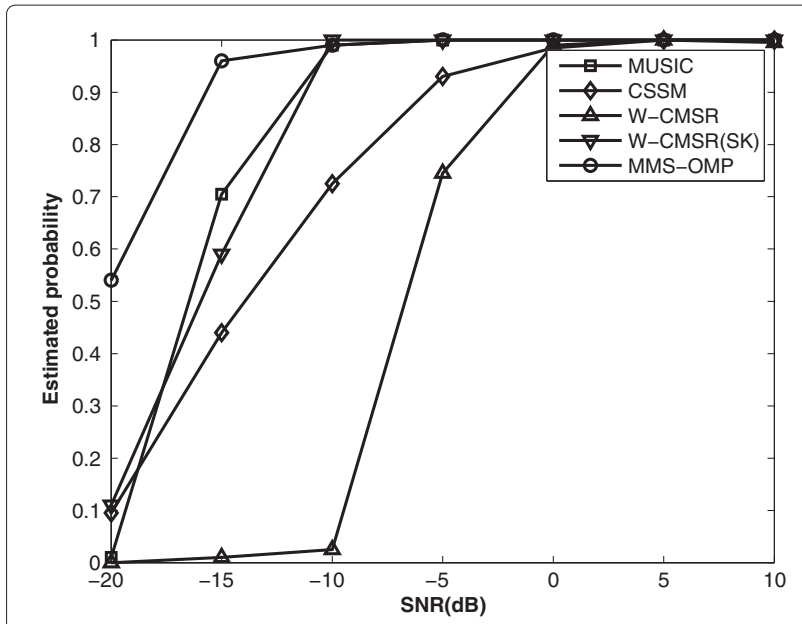

Figure 8 The estimated probability of the four algorithms versus SNR. The estimated probability is defined by the formula $E P=\frac{\text { count }}{\text { Mon }}$, where count is the number of successful estimation. If the DOAs are successfully estimated in one simulation, count $=$ count +1 . The settings are: $\theta_{1}=10^{\circ}, \theta_{2}=30^{\circ}, N=10$ sensors, $T=1,200$ time snapshots. The number of Monte-Carlo simulations is Mon $=500$.

varies from -20 to $10 \mathrm{~dB}$ as shown in Figure 8. Furthermore, we make a comparison between W-CMSR and MMS-OMP under the circumstances of the incident signals sharing different types of modulations. One incident signal is a BPSK signal with central frequency $9 \mathrm{MHz}$ and bandwidth of $2 \mathrm{MHz}$, the other one is formed as mixture of sinusoids with central frequency $11 \mathrm{MHz}$ and bandwidth of $2 \mathrm{MHz}$. The estimated performance of W-CMSR

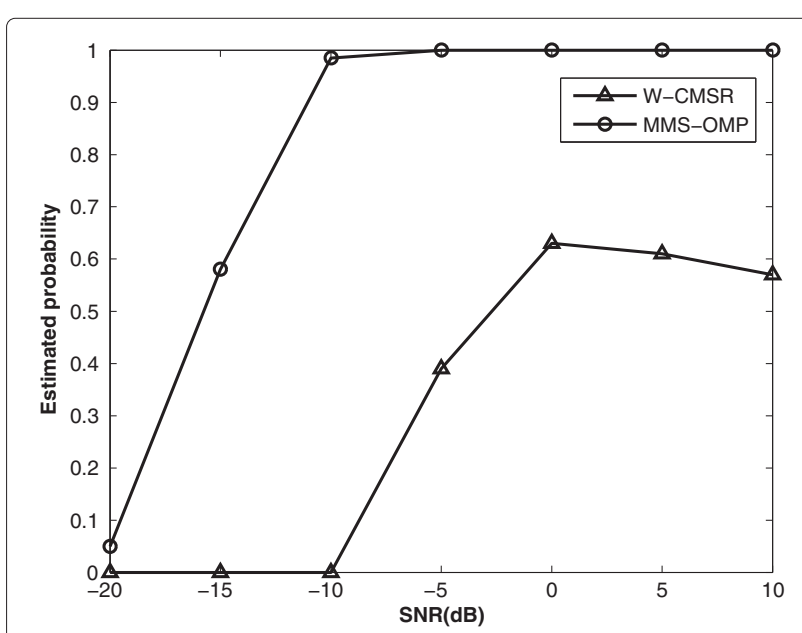

Figure 9 The estimated probability of W-CMSR and MMS-OMP when the two sources share different types of modulations. The only difference of simulation conditions between Figure 9 and Figure 8 is that the two sources share different types of modulations and bandwidths. One incident signal is a BPSK signal with central frequency $9 \mathrm{MHz}$ and bandwidth of $2 \mathrm{MHz}$, the other one is formed as mixture of sinusoids with central frequency $11 \mathrm{MHz}$ and bandwidth of $2 \mathrm{MHz}$. 
Table 1 Computation time comparison

\begin{tabular}{|c|c|c|c|c|c|}
\hline \multirow{2}{*}{ Band width } & \multirow{2}{*}{ Snapshot number } & \multicolumn{4}{|c|}{ Times (sec) } \\
\hline & & MUSIC & CSSM & W-CMSR & MMS-OMP \\
\hline \multirow{3}{*}{$20 \%$} & 80 & 0.0349 & 0.0125 & 1.8870 & 0.1997 \\
\hline & 320 & 0.0373 & 0.0153 & 1.9040 & 0.2019 \\
\hline & 1280 & 0.0421 & 0.0217 & 3.1497 & 0.2061 \\
\hline \multirow{3}{*}{$40 \%$} & 80 & 0.0546 & 0.0126 & 1.7160 & 0.3011 \\
\hline & 320 & 0.0562 & 0.0158 & 2.0090 & 0.3014 \\
\hline & 1280 & 0.0668 & 0.0234 & 2.7909 & 0.3083 \\
\hline \multirow{3}{*}{$80 \%$} & 80 & 0.0855 & 0.0127 & 1.8190 & 0.4329 \\
\hline & 320 & 0.0911 & 0.0168 & 2.1241 & 0.4402 \\
\hline & 1280 & 0.1023 & 0.0271 & 3.0339 & 0.4547 \\
\hline
\end{tabular}

is deteriorated just as illustrated in the introduction. The proposed method surpasses the W-CMSR algorithm by the absolute superiority in Figure 9, because our method relies less on a priori information of the incident signals than that of the W-CMSR algorithm.

Finally, the average computation time of those four methods are compared in different snapshots and signal bandwidths in the simulation V. In order to get a more conveniently adjustable bandwidth, we assume that two $0 \mathrm{~dB}$ signals which are formed as mixtures of equal-power sinusoids impinge onto the array from directions of $10^{\circ}$ and $30^{\circ}$ simultaneously. The signal bandwidth is set at $20,40,80 \%$, and the time domain snapshots are set at 80,320 and 1,280. For each bandwidth-snapshot pair, 500 Monte-Carlo trials are carried out to obtain the average computation time of those four methods, and the results are given in Table 1. The results show that MUSIC and CSSM are the two fastest methods. W-CMSR consumes large amount of computation time although its computational efficiency is not affected by the signal bandwidth. And MMS-OMP outperforms W-CMSR significantly in computational efficiency. Although MMS-OMP has larger computation time than the conventional methods, it is more important to note that it performs much better in DOA estimation.

\section{Conclusion}

In this article, we are engaged in the DOA estimation of wideband signal sources. We stack all the array covariance matrices, which are derived from passing the observed signal through a bank of narrowband filters, in the form of 3-D tensor matrix to put forward the slice-SR model in the frequency domain. We formulize the corresponding algorithm called MMS-OMP algorithm. This algorithm processes the array covariance data of each frequency bin jointly. The DOA estimation is achieved by jointly finding the sparsest coefficients of the MMS under the basis matrices. Its superiority embodies not only in the capability to resolve coherent sources, but also to incorporate prior information on the frequency spectra of the sources. Furthermore, the MMS-OMP is very efficient in relax the aliasing effects when the distance between adjacent array element is larger than the half-wavelength with respect to the highest frequency. The proposed method and the representative methods are compared. Simulation results show that our method has better noise robustness, higher resolution, and smaller computational complexity.

\section{Competing interests}

The authors declare that they have no competing interests.

Received: 6 July 2012 Accepted: 3 December 2012

Published: 12 February 2013

\section{References}

1. MA Doron, AJ Weiss, Maximum likelihood localization of wideband sources. IEEE Inte. Conf. Acoust. Speech Signal Process. 2, 489-492 (1992)

2. G Su, M Morf, The signal subspace approach for multiple wideband emitter location. IEEE Trans. Acoust. Speech Signal Process. 31(6), 1502-1522 (1983)

3. H Wang, M Kaveh, Coherent signal-subspace processing for the detection and estimation of angles of arrival of multiple wideband sources. IEEE Trans. Acoust. Speech Signal Process. 33(4), 823-831 (1985)

4. $\mathrm{M} \mathrm{H}$ Hung, Kaveh, Focussing matrices for coherent signal-subspace processing. IEEE Trans. Acoust. Speech Signal Process. 36(8), 1272-1281 (1988)

5. S Valaee, $\mathrm{P}$ Kabal, Wideband array processing using a two-sided correlation transformation. IEEE Trans. Signal Process. 43(1), 160-172 (1995)

6. MA Doron, AJ Weiss, On focusing matrices for wide-band array processing. IEEE Trans. Signal Process. 40(6), 1295-1302 (1992)

7. JJ Fuchs, On the application of the global matched filter to DOA estimation with uniform circular arrays. IEEE Inte. Conf. Acoust. Speech Signal Process. 49(4), 702-709 (2001)

8. D Malioutov, M Cetin, AS Willsky, A sparse signal reconstruction perspective for source localization with sensor arrays. IEEE Trans. Signal Process. 53(8), 3010-3022 (2005)

9. MM Hyder, K Mahata, Direction-of-arrival estimation using a mixed L2,0 norm approximation. IEEE Trans. Signal Process. 58(9), 4646-4655 (2010)

10. ZM Liu, ZT Huang, YY Zhou, Direction-of-arrival estimation of wideband signals via covariance matrix sparse representation. IEEE Trans. Signal Process. 59(9), 4256-4270 (2011)

11. JA Tropp, AC Gilbert, Signal recovery from random measurements via orthogonal matching pursuit. IEEE Trans. Inf. Theory. 53(12), 4655-4666 (2007) 
12. SF Cotter, BD Rao, E Kjersti, K Kreutz-Delgado, Sparse solutions to linear inverse problems with multiple measurement vectors. IEEE Trans. Signal Process. 53(7), 2477-2488 (2005)

13. M Wax, T Kailath, Detection of signals by information theoretic criteria. IEEE Trans. Acoust. Speech Signal Process. 33(2), 387-392 (1985)

14. DM Malioutov, A sparse signal reconstruction perspective for source localization with sensor arrays. PhD thesis, Massaschusetts Institute of Technology, Department of Electrical Engineering and Computer Science (2003)

15. E Fishler, A Haimovich, RS Blum, LJ Cimini, D Chizhik, RA Valenzuela, Spatial diversity in radars-models and detection performance. IEEE Trans. Signal Process. 54(3), 823-838 (2006)

doi:10.1186/1687-6180-2013-18

Cite this article as: Gan and Wang: DOA estimation of wideband signals based on slice-sparse representation. EURASIP Journal on Advances in Signal Processing 2013 2013:18.

\section{Submit your manuscript to a SpringerOpen ${ }^{\mathcal{O}}$ journal and benefit from:}

- Convenient online submission

- Rigorous peer review

- Immediate publication on acceptance

- Open access: articles freely available online

- High visibility within the field

- Retaining the copyright to your article

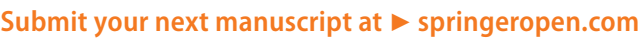

